\title{
Uso de diferentes tipos de calçados não interfere na postura ortostática de mulheres hígidas
}

\section{Use of different types of shoes do not interfere in bealthy women orthostatic posture}

\author{
Geraldo Fabiano de Souza Moraes ${ }^{[a]}$, Adriana Papini Antunes ${ }^{[b]}$, Elaine Souza Rezende ${ }^{[c]}$, \\ Poliana Cardoso Ribeiro de Oliveira ${ }^{[\mathrm{d}]}$
}

[a] Fisioterapeuta, Mestre em Ciências da Reabilitação, Especialista em Fisioterapia Ortopédica e Esportes, professor adjunto e coordenador do curso de Fisioterapia do Centro Universitário Newton Paiva, Belo Horizonte, MG - Brasil, e-mail: geraldmoraes@terra.com.br

[b] Fisioterapeuta, Especialista em Fisioterapia Ortopédica e Desportiva, Centro Universitário Newton Paiva, Belo Horizonte, MG Brasil, e-mail: dripantunes@yahoo.com.br

[c] Fisioterapeuta, Especialista em Fisioterapia Ortopédica e Desportiva, Centro Universitário Newton Paiva, Belo Horizonte, MG Brasil, e-mail: elainerezende@gmail.com

[d] Fisioterapeuta, Centro Universitário Newton Paiva, Belo Horizonte, MG - Brasil, e-mail: polianacro@yahoo.com.br

\section{Resumo}

Introdução: Nos últimos anos, as mulheres passaram a usar sapatos de salto para ficarem mais bonitas. Entretanto, tal hábito desencadeou vários efeitos negativos para o sistema musculoesquelético. Um dos aspectos mais prevalentes são queixas de mulheres com desconforto na região lombar durante o uso de sapato de salto. Autores descreveram que pode haver relação entre a postura assumida e os desconfortos presentes, geralmente associados à fadiga muscular e ao aumento das forças de reação do solo durante o uso desse tipo de calçado. Existem controvérsias na literatura a respeito do efeito do uso de sapatos de salto por mulheres nas alterações posturais e sobrecarga sobre a coluna vertebral e segmentos corporais. Objetivo: O objetivo do presente estudo foi verificar se diferentes tipos de calçados são capazes de alterar significativamente a biomecânica estática a partir da avaliação postural. Metodologia: Quinze mulheres sedentárias foram submetidas a uma avaliação postural estática com a utilização do programa Software para Análise Postural. Análise de variância para medidas repetidas foi utilizada para investigar diferenças entre os calçados em todas as variáveis posturais analisadas. O nível de significância estabelecido foi de $\alpha$ igual a 0,05 . Resultados: Os resultados mostraram que não houve diferença significativa na postura estática com os tipos de calçados e descalço. Conclusão: Conclui-se que os calçados não interferiram na postura estática da amostra.

Palavras-chave: Postura. Avaliação. Biomecânica. 


\begin{abstract}
Introduction: In the last years women began to wear high-heeled shoes to become more beautiful. However, this habit also triggered several negative effects on the musculoskeletal system. One of the most prevalent aspects is women with complaints of discomfort in the lumbar region for the use of high-heeled shoe. Authors described that there may be a relation between attitude and the present discomfort, often associated with muscular fatigue and increase the ground reaction forces during the use of such footwear. There are controversies in the literature about the effect of using high-heeled shoes for women in postural changes and overload on the spine and body segments. Objective: The purpose of this study was to verify if different types of shoes are able to significantly modify the biomechanics through static postural evaluation. Methodology: Fifteen sedentary women were subjected to a static evaluation posture with the use of the Software for Postural Evaluation. Variance analysis for repeated measures was used to investigate differences between the shoes in all variables postural analyzed. The significance level was $\alpha$ equal 0,05. Results: The results showed no significant difference in static position with the types of shoes and barefoot. Conclusion: It was concluded that the shoes did not interfere in static position of this sample.
\end{abstract}

Keywords: Posture. Evaluation. Biomechanics.

\title{
Introdução
}

Desde o reinado de Luís XIV na França, as mulheres passaram a usar sapatos de salto para ficarem mais bonitas. Entretanto, tal hábito desencadeou vários efeitos negativos para o sistema musculoesquelético (1). De acordo com Esenyel et al. (2), 37\% a 69\% das mulheres usam salto alto no dia a dia, o que pode, em alguns casos, levar à diminuição da função dos membros inferiores (MMII) e, consequentemente, desordem na marcha (3).

Em relação ao desencadeamento de incapacidades - que é caracterizada como resultado de uma relação entre o estado ou condição de saúde do indivíduo e as circunstâncias nas quais o indivíduo vive (4) -, vários são os relatos associados ao tipo de calçado utilizado, à tarefa executada e ao contexto envolvido (5).

Snow e Williams (6) descreveram que pode haver uma relação entre a postura assumida e os desconfortos presentes, geralmente associados à fadiga muscular e ao aumento das forças de reação do solo durante o uso desse tipo de calçado. Um dos aspectos mais prevalentes são queixas de mulheres com desconforto na região lombar durante o uso de sapato de salto (5).

Existe uma controvérsia na literatura a respeito do efeito do uso de sapatos de salto nas alterações posturais e sobrecarga sobre a coluna vertebral e segmentos corporais.

Opila et al. (7) demonstraram que o uso de sapato de salto, comparado com a condição descalça, causa uma retificação da região lombar. Uma explicação para este fato seria um encurtamento por contração isométrica da cadeia posterior, que gera retificação da coluna lombar, e pela tentativa do sistema em compensar a inclinação anterior do tronco que está associada ao uso de calçados de salto alto. Outro fator a se considerar é que, para reposicionar o centro de gravidade do corpo mais posteriormente e aliviar a sensação de cair para frente, ocorre uma cocontração dos músculos abdominais e paravertebrais.

Lateur et al. (8) relataram que o uso do sapato de salto provoca diminuição da curvatura lombar. Isto pode ser explicado pelo fato do centro de gravidade do indivíduo não mudar significativamente quando este está com salto alto ou salto baixo, o que pode indicar a existência de uma compensação anatômica nos MMII e na região lombar da coluna vertebral para manter o corpo em equilíbrio.

Snow e Williams (6) relataram que não foi encontrada diferença significativa na média da curvatura lombar ou na inclinação pélvica entre as alturas do salto.

Frey et al. (9) encontraram que $75 \%$ das mulheres que utilizavam salto alto apresentavam um ou mais deformidades no antepé, sendo $71 \%$ hálux valgo, $50 \%$ dedos em martelo, $18 \%$ joanete do $\mathrm{V}$ metatarso, $13 \%$ proeminência da cabeça do metatarso e 4\% apresentavam várias deformidades. 
Kerrigan, Todd e Riley (10) estudaram a influência dos saltos grosso e fino e concluíram que caminhar com saltos altos aumenta a força por meio da articulação patelofemural e de compressão no compartimento medial do joelho, e Kerrigan, Lelas e Karvosky (11) descreveram que os saltos altos e grossos causam forças anormais sobre os compartimentos mediais dos joelhos, o que pode predispor a alterações degenerativas dos joelhos.

O uso do salto alto na postura ortostática implica retroversão pélvica, aproximação dos joelhos e tornozelos em relação à linha de gravidade, deslocamento posterior da cabeça e da coluna torácica. A utilização do salto alto submete o joelho a torques excessivos, principalmente em varo, o que torna a porção medial desta articulação mais suscetível a transformações degenerativas da cartilagem articular, e, assim, está relacionado à maior incidência de osteoartrite (12).

A utilização de sapatos de salto alto submete o corpo a condições não fisiológicas que necessitam de constantes adaptações e acomodações. A função das articulações é alterada, o que pode desencadear forças e torques acima do normalmente exercido. Além disso, demanda que alterações posturais sejam feitas a fim de manter o equilíbrio postural (13).

Entre os melhores recursos para a prevenção está em optar pelos calçados mais baixos, que dão maior sustentação e equilíbrio, e não forçam determinadas áreas do corpo. O mais indicado pelos médicos é fazer, durante a semana, um rodízio entre saltos, não deixando que os pés se acostumem a um tipo específico de salto. Outra boa prática é alongar periodicamente os músculos da coxa e da panturrilha (14).

Nos últimos anos, distúrbios osteomusculares relacionados ao trabalho (DORT) e acidentes de trabalho têm preocupado dirigentes de empresas e funcionários, principalmente por causa do absenteísmo, diminuição da produtividade e diminuição de rendimentos. Os profissionais acometidos por estes problemas precisam manter-se afastados de suas atividades profissionais por um determinado período. DORT são problemas que podem ocorrer quando a pessoa usa demais uma determinada parte do corpo, repetindo ou mobilizando gestos, o que causa um sofrimento local nas articulações (15).

O salto alto pode causar à saúde de suas usuárias lesões, deformações e doenças, além de estar associada a riscos ocupacionais (15). Segundo a $1^{\text {a }}$ Turma do Tribunal Regional do Trabalho da $2^{\text {a }}$ Região do Estado de São Paulo, a empresa deve cuidar para que o empregado não utilize roupas ou sapatos que coloquem em risco sua integridade física, o que faz com que lesões relacionadas ao uso de salto alto possam ser considerada acidente de trabalho (16).

Hoje em dia, as mulheres estão mais inseridas no mercado de trabalho e o uso de salto é frequente, principalmente associado à questão estética. Pouco se tem estudado sobre a influência dos calçados nas articulações dos MMII e na postura. Graças a relatos de incômodos como dores, alterações posturais e deformidades nos pés, é extremamente importante verificar qual tipo de calçado seria o mais adequado e o mais confortável para o uso diário.

Portanto, o objetivo do presente estudo foi verificar se diferentes tipos de calçados são capazes de alterar significativamente a biomecânica estática, por meio da avaliação postural.

\section{Materiais e métodos}

\section{Delineamento do estudo e caracterização da amostra}

Trata-se de um estudo observacional transversal, no qual foi utilizado uma amostra de conveniência com 15 mulheres jovens, com idades entre 18 e 35 anos (23,2 \pm 3,36 anos), sedentárias. O tempo de uso dos calçados/semana está demonstrado no Gráfico 1. A variação do uso dos calçados em horas/dia está demonstrada no Gráfico 2. O número do calçado variou de 36 a 37 (36 0 0,81). Das 15 voluntárias, 13 eram destras e duas eram canhotas.

Os critérios de exclusão foram mulheres que possuíssem rigidez articular, dor que incapacitasse a realização dos testes, discrepância de MMII (acima de $1 \mathrm{~cm}$ ), terem tido entorse de tornozelo há menos de seis meses, terem sido diagnosticadas com ruptura ligamentar nos tornozelos, terem sido submetidas a cirurgias ou fisioterapia nos membros inferiores há menos de seis meses. Mulheres obesas - IMC $\geq 30$ (17) -, grávidas, 
diabéticas, que utilizassem auxílio à marcha e/ou palmilhas, presença de edema de tornozelo e pés, hipotrofia acima de $2 \mathrm{~cm}$ de panturrilha e coxa, e que possuíssem hálux valgo, joanete do $\mathrm{V}$ metatarso e neuroma de Morton que impossibilitasse a realização dos procedimentos.

Este estudo foi submetido e aprovado pelo Comitê de Ética e Pesquisa do Centro Universitário Newton Paiva, Parecer n. 0082, de 6 de março de 2008.

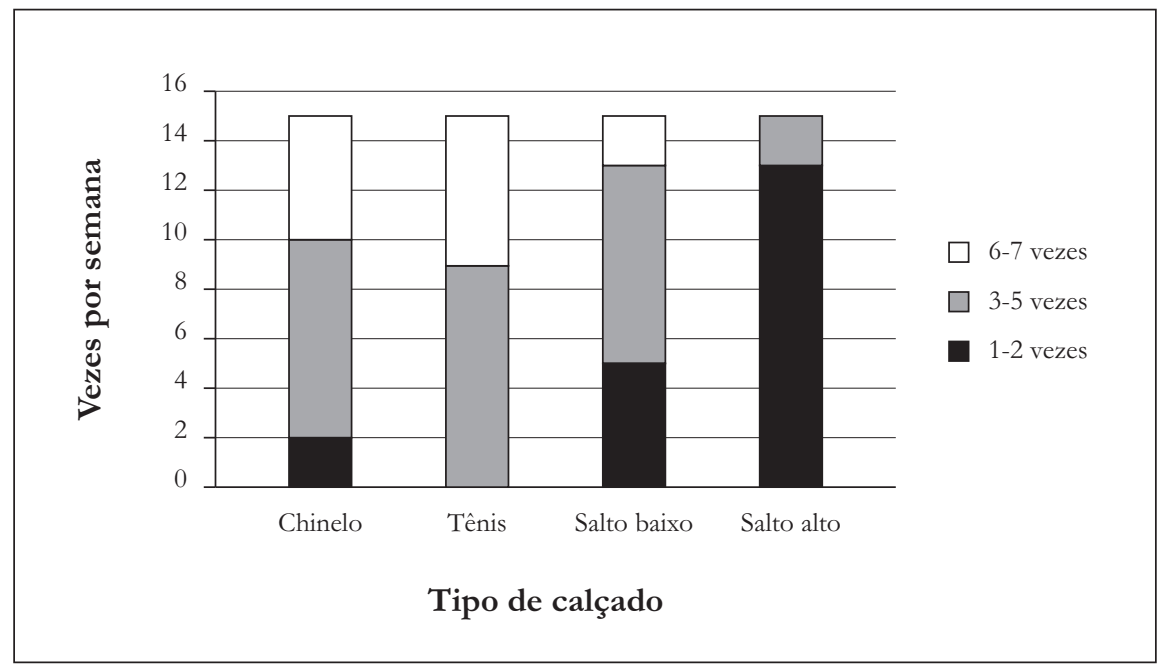

Gráfico 1 - Tempo de uso dos calçados por semana

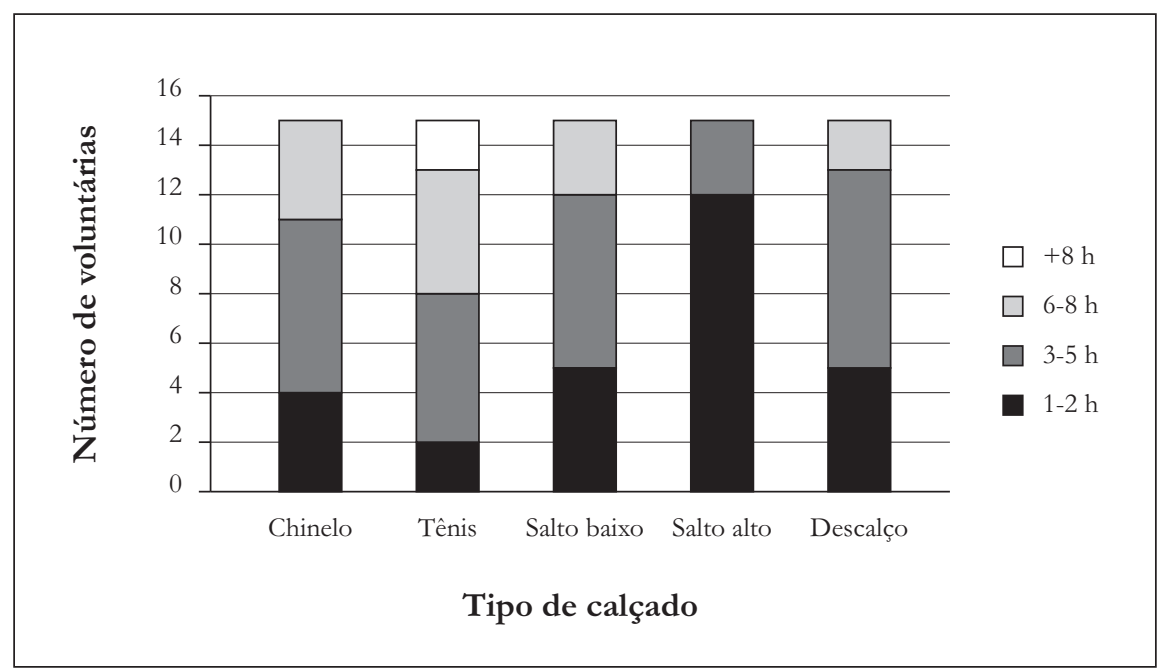

Gráfico 2 - Variação do uso dos calçados em horas por dia

\section{Instrumentos}

\section{Goniômetro universal}

As mensurações da flexibilidade foram feitas com um goniômetro universal (Baseline ${ }^{\circledR}$, Aurora, IL, EUA), tendo duas hastes de $31,5 \mathrm{~cm}$ de comprimento e um fulcro com precisão de medida de um grau. A goniometria refere-se à medida dos ângulos formados nas articulações pelos segmentos corporais. Ao utilizar um goniômetro 
universal, o examinador obtém essas medidas colocando as partes do instrumento (braço fixo, fulcro e braço móvel) junto aos segmentos imediatamente proximal e distal à articulação que está sendo avaliada. É um equipamento de medida do ângulo articular que apresenta validade e confiabilidade já descritas na literatura $(18,19)$.

A goniometria pode ser utilizada para determinar tanto uma posição articular específica quanto sua amplitude total de movimento.

\section{Programa para avaliação postural}

Foi utilizado o programa Software for Posture Evaluation - Software para Avaliação Postural (SAPO, Incubadora Fapesp, SP, Brasil). É um programa de computador que, a partir de fotografias digitalizadas do indivíduo, permite a mensuração da posição, comprimento, ângulo e alinhamento, entre outras propriedades, dos segmentos corporais de um indivíduo, o que permite uma análise postural estática nos planos frontal (vista anterior e posterior - Figuras 1a e 1b) e sagital (vista direita e esquerda - Figura 1c e 1d).
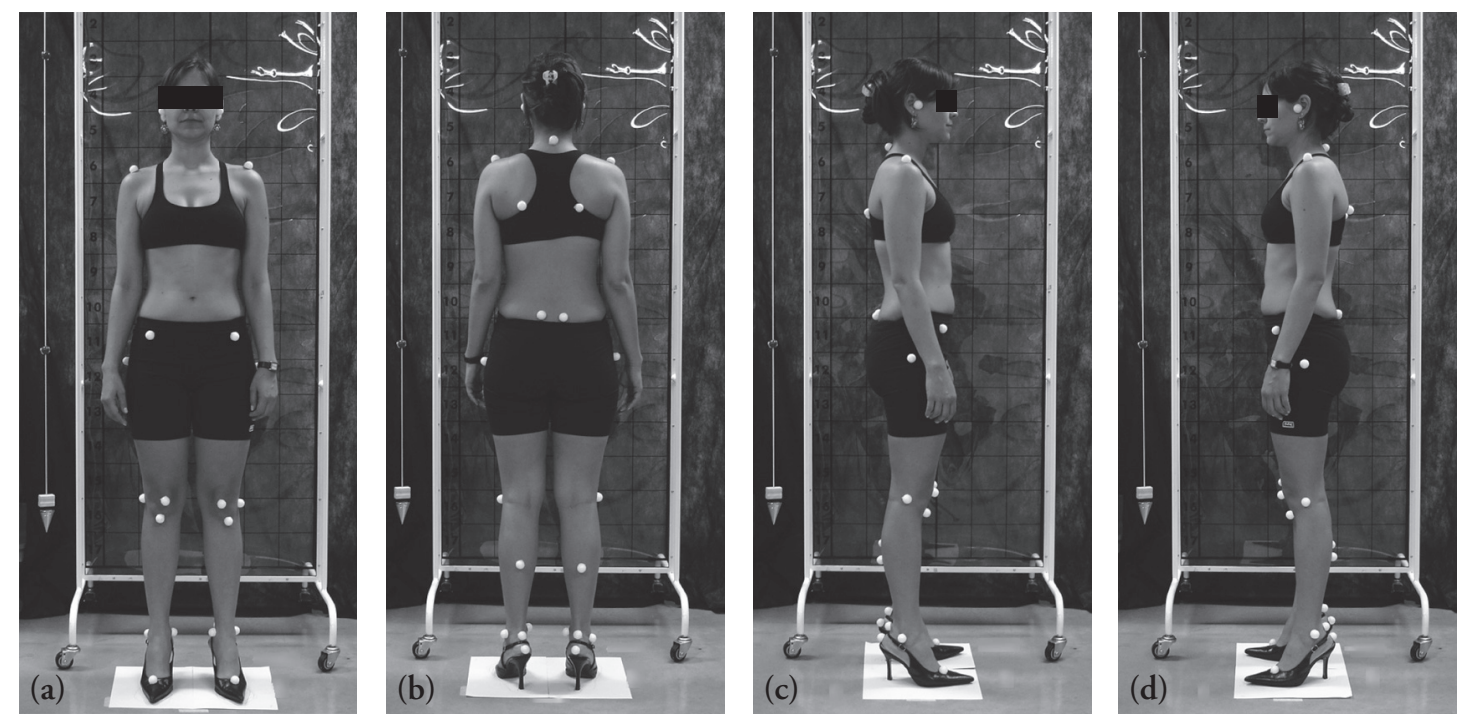

Figura 1 - Análise postural estática com o programa SAPO - (a) vista anterior, (b) vista posterior, (c) vista lateral direita e (d) vista lateral esquerda

Braz, Goes e Carvalho (20), em seu estudo, demonstraram a validade e confiabilidade do programa ao realizar medidas repetidas por três avaliadores sem obter diferenças substanciais. Ferreira (21) demonstrou que a confiabilidade do programa depende da marcação correta dos pontos anatômicos e a colocação de marcadores nos pontos anatômicos permite maior confiabilidade.

Neste estudo, a confiabilidade intraexaminador foi medida pelo Coeficiente de Correlação Intraclasse (CCI) seguindo o modelo 3, com o objetivo de relatar a reprodutibilidade das medidas a serem utilizadas pelo estudo. O valor obtido foi CCI $(3,1)$ igual a 0,91 para a condição descalça; 0,89 para a condição chinelo; 0,93 para a condição tênis; 0,89 para a condição salto baixo e 0,88 para a condição salto alto.

\section{Procedimentos}

Inicialmente, todas as voluntárias foram esclarecidas quanto aos objetivos e procedimentos do estudo e, em seguida, assinaram o termo de consentimento livre e esclarecido. Em seguida, foi feita uma avaliação 
padronizada, por uma avaliadora devidamente treinada, para identificação, anamnese e exame físico de cada voluntária. No exame físico, foi feita a goniometria de dorsiflexão, bilateralmente.

Posteriormente, foi realizado o registro da postura estática da voluntária na posição ortostática, nos planos frontal (vista anterior e posterior) e sagital (direito e esquerdo) por meio de câmera digital modelo Sony Cybershot DSC-P200 (Sony Electronics Inc.C, San Diego, CA), seguindo a seguinte ordem de calçados: descalço, chinelo, salto baixo, salto alto e tênis. As fotos foram armazenadas para posterior análise no programa SAPO.

Os sapatos utilizados foram: salto alto tipo Chanel ${ }^{\circledR}$ (Figura 2a), salto baixo tipo Chanel ${ }^{\circledR}$ (Figura 2b), tênis (Figura 2c), chinelo modelo Havaianas ${ }^{\circledR}$ (Figura 2d) e descalço. Foram considerados sapato de salto alto aqueles com altura entre 9 e $11 \mathrm{~cm}$ e para salto baixo, aquele entre 5 e $7 \mathrm{~cm}$. Para o tênis foi necessário este apresentasse elevação de calcanhar de 2 a 4 cm.
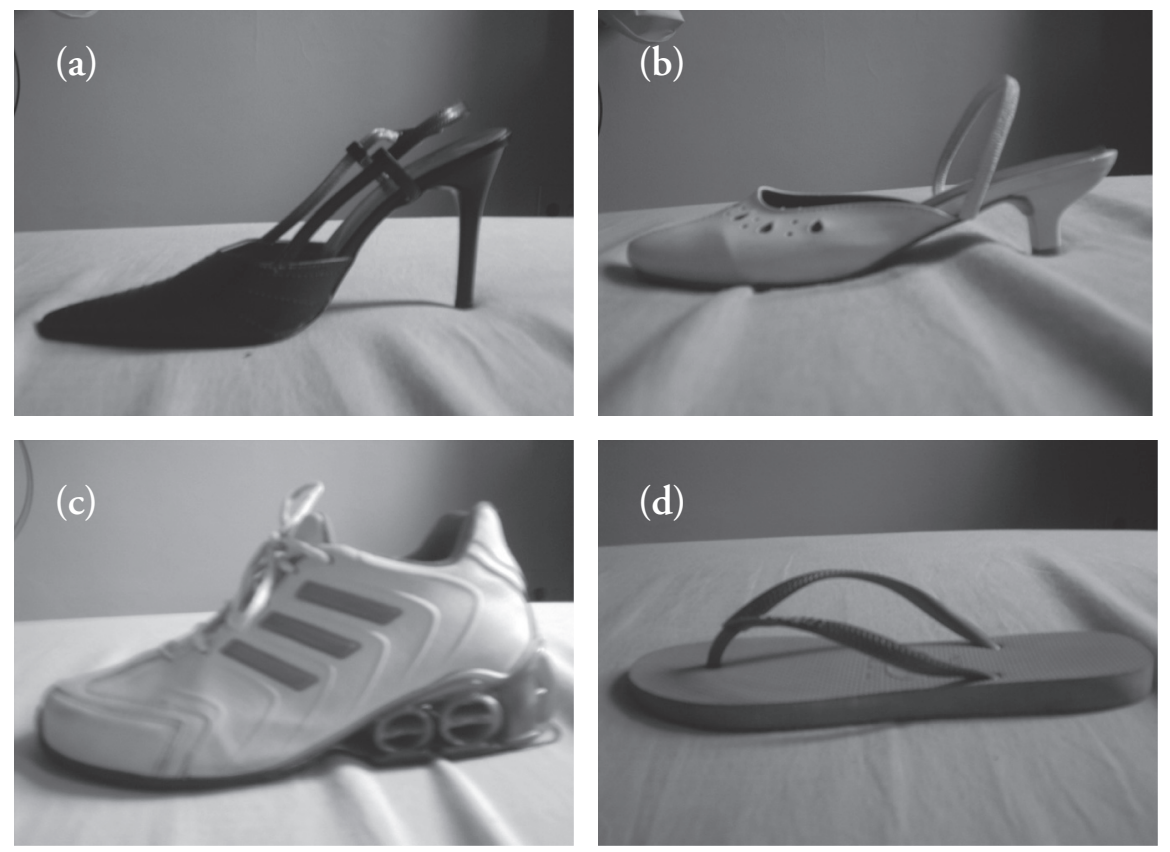

Figura 2 - Tipos de calçados utilizados - (a) salto alto tipo Chanel, (b) salto baixo tipo Chanel, (c) tênis, (d) chinelo modelo Havaianas ${ }^{\circledR}$

\section{Redução dos dados}

Para avaliação da postura estática, utilizou-se o programa SAPO e as variáveis utilizadas foram: alinhamento horizontal da cabeça (AHC), alinhamento vertical da cabeça (AVCA), alinhamento vertical do tronco (AVT), ângulo do quadril (AQ), alinhamento vertical do corpo (AVCO), alinhamento horizontal da pelve (AHP), ângulo do joelho (AJ), ângulo do tornozelo (AT), centro de gravidade: assimetria do plano frontal (APF) e assimetria do plano sagital (APS). Todos os valores correspondentes às variáveis analisadas foram obtidos seguindo as recomendações do programa para análise da postura nas respectivas condições estudadas.

\section{Análise estatística}

Análise de variância (ANOVA) para medidas repetidas foi utilizada para investigar diferenças estatisticamente significativas entre os calçados em todas as variáveis analisadas do SAPO. Foi utilizado o pacote 
estatístico Statistical Package of Social Science for Windows (SPSS) na sua versão15.0. (SPSS IncC., Chatanoga, IL). A significância estabelecida foi de $\alpha$ igual 5\%.

\section{Resultados}

\section{Avaliação postural}

Para a avaliação postural utilizando o SAPO, não foi apresentada diferença significativa para todas as variáveis avaliadas na vista lateral direita e esquerda: AHCD ( $p=0,822)$, AHCE $(p=0,651), \operatorname{AVCAD}(p=0,713)$, $\operatorname{AVCAE}(p=0,998), \operatorname{AVTD}(p=0,785), \operatorname{AVTE}(p=0,387), A Q D(p=0,846), A Q E(p=0,489), \operatorname{AVCOD}(p=0,059)$, $\operatorname{AVCOE~}(p=0,076), \operatorname{AHPD}(p=0,909), \operatorname{AHPE}(p=0,906), \operatorname{AJD}(p=0,722), \operatorname{AJE}(p=0,390), \operatorname{ATD}(p=0,399)$, ATE $(p=0,453)$. Para as variáveis relacionadas ao centro de gravidade não houve diferenças significativas: APF $(p=0,291)$ e APS $(p=0,339)$. Os valores de média e desvio padrão dos itens avaliados encontram-se na Tabela 1.

Tabela 1 - Médias e desvios padrão das variáveis avaliadas

\begin{tabular}{lcccccc}
\hline Ângulo & Lado & Descalço & Chinelo & Tênis & Salto baixo & Salto alto \\
\hline \multirow{2}{*}{ AHC } & D & $50,28 \pm 4,63$ & $51,26 \pm 4,59$ & $52,44 \pm 5,36$ & $50,48 \pm 6,28$ & $50,63 \pm 6,37$ \\
& E & $52,54 \pm 5,04$ & $53,18 \pm 4,58$ & $51,93 \pm 4,16$ & $54,40 \pm 4,41$ & $53,12 \pm 4,30$ \\
AVCA & D & $12,97 \pm 5,98$ & $13,43 \pm 6,81$ & $11,32 \pm 7,79$ & $14,98 \pm 6,98$ & $12,32 \pm 7,49$ \\
& E & $14,97 \pm 5,66$ & $14,87 \pm 4,63$ & $15,58 \pm 6,12$ & $14,99 \pm 7,34$ & $15,15 \pm 5,45$ \\
AVT & D & $-1,44 \pm 2,67$ & $-1,00 \pm 2,38$ & $-0,70 \pm 1,82$ & $-1,20 \pm 2,45$ & $-1,72 \pm 2,24$ \\
& E & $-2,92 \pm 1,71$ & $1,09 \pm 14,65$ & $-2,29 \pm 1,92$ & $-3,36 \pm 1,98$ & $-2,79 \pm 2,41$ \\
AQ & D & $-8,17 \pm 5,30$ & $-6,96 \pm 5,77$ & $-6,16 \pm 3,75$ & $-6,56 \pm 5,80$ & $-7,40 \pm 4,71$ \\
& E & $-8,48 \pm 3,84$ & $-7,64 \pm 3,72$ & $-6,63 \pm 3,58$ & $-8,14 \pm 3,66$ & $-6,36 \pm 4,35$ \\
AVCO & D & $2,16 \pm 1,09$ & $1,86 \pm 1,45$ & $2,07 \pm 1,24$ & $1,52 \pm 1,13$ & $0,89 \pm 1,48$ \\
& E & $0,98 \pm 1,21$ & $1,16 \pm 1,16$ & $1,02 \pm 1,05$ & $0,23 \pm 1,55$ & $0,17 \pm 1,10$ \\
AHP & D & $-12,36 \pm 4,27$ & $-12,04 \pm 5,85$ & $-13,26 \pm 5,00$ & $-11,74 \pm 5,48$ & $-11,53 \pm 5,47$ \\
& E & $-13,69 \pm 4,37$ & $-14,25 \pm 4,43$ & $-14,84 \pm 5,58$ & $-13,50 \pm 6,03$ & $-13,02 \pm 6,16$ \\
AJ & D & $-3,54 \pm 4,34$ & $-3,02 \pm 4,13$ & $-2,05 \pm 3,78$ & $-3,64 \pm 4,47$ & $-4,12 \pm 4,43$ \\
& E & $-4,21 \pm 5,27$ & $-1,77 \pm 4,35$ & $-1,22 \pm 4,13$ & $-3,24 \pm 4,36$ & $-1,98 \pm 4,81$ \\
AT & D & $86,82 \pm 3,24$ & $87,07 \pm 2,90$ & $86,74 \pm 3,03$ & $88,19 \pm 3,13$ & $88,48 \pm 3,22$ \\
APF & E & $87,27 \pm 3,61$ & $86,72 \pm 3,43$ & $86,88 \pm 3,26$ & $88,46 \pm 3,65$ & $88,42 \pm 2,80$ \\
& NA & $-0,017 \pm 4,58$ & $-0,007 \pm 4,68$ & $-0,070 \pm 16,06$ & $0,023 \pm 5,72$ & $0,017 \pm 5,71$ \\
& NA & $0,314 \pm 13,95$ & $0,305 \pm 14,12$ & $0,265 \pm 11,63$ & $0,228 \pm 13,99$ & $0,247 \pm 13,21$ \\
\hline
\end{tabular}

$\mathrm{AHC}=$ alinhamento horizontal da cabeça; $\mathrm{AVCA}=$ alinhamento vertical da cabeça; $\mathrm{AVT}=$ alinhamento vertical do tronco; $\mathrm{AQ}=$ ângulo do quadril; $\mathrm{AVCO}=$ alinhamento vertical do corpo; $\mathrm{AHP}=$ alinhamento horizontal da pelve; $\mathrm{AJ}=$ ângulo do joelho; $\mathrm{AT}=$ ângulo do tornozelo; $\mathrm{APF}=$ assimetria do plano frontal; $\mathrm{APS}=$ assimetria do plano sagital; $\mathrm{D}=$ Direito; $\mathrm{E}=\mathrm{Esquerdo} ; \mathrm{NA}=$ não se aplica; Média \pm sd. 


\section{Discussão}

\section{Avaliação postural}

Postura pode ser considerada a posição assumida pelos segmentos corporais durante as atividades de vida diária (AVD) realizadas por um indivíduo (22). Uma postura adequada pode ser considerada aquela em que o corpo encontra-se em condição favorável à realização da tarefa (23).

Estudos têm descrito a influência do salto alto nas adaptações da locomoção dos indivíduos $(13,24)$. Durante a análise dinâmica da locomoção, diversas adaptações são necessárias para acomodação do aparato do indivíduo às condições a que o sistema está sendo submetido, bem como as influências do contexto em que a tarefa está sendo realizada $(25,26)$.

No presente estudo, a partir da avaliação da postura estática com os diversos calçados e na condição descalça não se observou diferenças significativas para todas as variáveis avaliadas. Na postura estática, a maioria das adaptações não corresponde às observadas na postura durante atividades dinâmicas (27). Tal fato pode ser justificado pela alteração na relação demanda-capacidade associada às condições nas quais a atividade está sendo realizada.

Diversos são os fatores que podem interferir no alinhamento do corpo e posicionamento dos segmentos corporais, tais como eficiência muscular e condições patológicas neurológicas ou musculoesqueléticas, presentes em condições rotineiras ou adversas às habituais.

Dessa forma, um dos fatores que podem ser ressaltados como influência direta na postura estática e dinâmica são as adaptações musculoesqueléticas decorrentes das condições de realização de uma determinada tarefa, bem como as compensações decorrentes desta.

Em relação ao uso de calçados, os músculos dos MMII podem encurtar-se, principalmente os músculos da cadeia posterior, como tríceps sural, ísquio surais, paravertebrais. As voluntárias tinham o hábito de usar mais calçados de salto negativo e descalço do que salto alto durante a semana, bem como passar mais horas com eles. Desta forma, curtos períodos de uso de calçados com o calcanhar elevado significativamente pouco podem ter interferido na postura das voluntárias durante a realização dos testes.

Mulheres que têm o hábito de usar salto alto regularmente, como secretárias, e de permanecer longos períodos na posição ortostática, como balconistas, tendem a desenvolver encurtamentos adaptativos à condição do uso de calçados e situação em que mantêm o corpo nas AVD e atividades profissionais (28). Algumas mulheres relatam dificuldades durante a locomoção pela presença de dor, principalmente nos tornozelos, e falseios quando mudam do calçado de salto alto para os calçados com menor inclinação (29).

Em relação à avaliação do centro de gravidade, por meio das variáveis APS e APF, não houve diferenças em relação às condições dos calçados e descalço. Lateur et al. (8) demonstraram que o uso de calçados de salto alto e baixo não modificou significativamente o centro de gravidade das voluntárias avaliadas. Graças às características anatômicas e antropométricas de mulheres, tais como o alargamento da pelve, alteração do ângulo quadriciptal e composição corpórea, o sistema frequentemente desenvolve compensações nos MMII e na região lombar.

Outro fator relatado na literatura é a condição de avaliação em laboratório, na qual o voluntário tem consciência das atividades a serem executadas, principalmente durante a avaliação e o registro da postura de forma estática (30).

Na prática clínica pode-se observar, frequentemente, relatos de dores na região lombar, dos quadris, joelhos e tornozelos. O uso esporádico de sapatos de salto alto pode ser considerado uma perturbação na qual o indivíduo necessitará encontrar estratégias adaptativas para que o sistema possa suportar a massa corporal com menor demanda muscular e, consequentemente, menor gasto energético. A incapacidade do sistema em adaptar-se às novas condições pode ser um fator relevante ao desenvolvimento de quadros álgicos e incidência de lesões nas articulações dos MMII, bem como a aceleração do processo degenerativo articular.

Casarin (31), em seu estudo com 14 mulheres com uso diário de salto alto e 10 com uso não diário de salto alto, analisou as alterações posturais e atividade eletromiográfica, de maneira estática, na posição ortostática dos músculos gastrocnêmio e eretores da espinha, nas condições descalço, calcanheira, salto baixo e salto alto, e não encontrou diferenças significativas no ângulo lombar de todas as voluntárias avaliadas. 


\section{Relevância clínica}

Não há na literatura grande volume de informações acerca da avaliação postural associada à avaliação da atividade muscular de MMII e da coluna lombar com o uso dos diversos calçados e descalço. Além disso, há controvérsias em alguns estudos, principalmente em relação aqueles que simulam a elevação do calcanhar, progressivamente, com aparatos de madeira e outros materiais.

O uso regular de salto, principalmente o alto, pode levar ao aprendizado motor da mulher que usa este calçado com maior frequência, pois a repetição de uma tarefa é um fator determinante para plasticidade cortical que, por sua vez, gera automatismo do gesto (32).

\section{Conclusão}

A utilização de diferentes calçados não interferiu na postura estática em ortostatismo com o uso de chinelo, tênis, salto baixo, salto alto e na condição descalça; desta forma, a análise do calçado isoladamente não pode ser considerada como único fator nas alterações posturais de mulheres.

\section{Referências}

1. Lee C, Jeong E, Freivalds A. Biomechanical effects of wearing high-heeled shoes. Int J Ind Ergonomics. 2001;28(6):321-26.

2. Esenyel M, Walsh K, Walden JG, Gitter A. Kinetics of high-heeled gait. J Am Podiatr Med Assoc. 2003;93(1):27-32.

3. Hong WH, Lee YH, Chen HC, Pei YC, Wu CY. Influence of heel height and shoe insert on comfort perception and biomechanical performance of young female adults during walking. Foot Ankle Int. 2005;26(12):1042-48.

4. Farias N, Buchalla C. A classificação internacional de funcionalidade, incapacidade e saúde da Organização Mundial da Saúde: conceitos, usos e perspectivas. Rev Bras Epidemiol. 2005;8(2):187-93.

5. Frey CMD. Foot health and shoewear for women. Clinical Orthopaedics and Related Research. 2000;372(3):32-44.

6. Snow R, Williams K. High heeled shoes: their effect on center of mass position, posture, three-dimensional kinematics, rear foot motion and ground reaction forces. Arch Phys Med Rehabil. 1994;75(5):568-76.

7. Opila KA, Wagner SS, Schiowitz S, Chen J. Postural alignment in barefoot and high-heeled stance. Spine. 1988;13(5):542-47.

8. Lateur BJ, Giaconi RM, Questad K, Ko M, Lehmann JF. Footwear and posture: compensatory strategies for heel height. Am J Phys Med Rehabil. 1991;70(5):246-54.

9. Frey C, Thompson F, Smith J, Sanders M, Horstman H. American Orthopaedic Foot and Ankle Society women's shoe survey. Foot \& Ankle. 1993;14(2):78-81.

10. Kerrigan D, Todd M, Riley P. Knee osteoarthritis and high-heeled shoes. Lancet. 1998;351(9113):1399-401.

11. Kerrigan D, Lelas J, Karvosky M. Women’s shoes and knee osteoarthritis. Lancet. 2001;357(9262):1097-98.

12. Sacco ICN, Melo MCS, Rojas GB, Naki IK, Burgi K, Silveira LTY, et al. Análise biomecânica e cinesiológica de posturas mediante fotografia digital: estudo de casos. Rev Brás Ciênc Mov. 2003;11(2):25-33.

13. Wannmacher, L. Osteoartrose de joelhos. Parte II: evidências sobre abordagens não-medicamentosas. Uso racional de medicamentos: temas selecionados. [revista de Internet] 2006 [acesso 22 fev. 2010];3(4). Disponível em: http:// portal.saude.gov.br/portal/arquivos/pdf/v3n4_osteoartrose_parte2.pdf

14. Potério-Filho J, Silveira SAF, Potério GMB, Junior RF, Almeida FHM, Menezes FH. The effect of walking with high-heeled shoes on the leg venous pressure. Angiology. 2006;57(4):424-30. 
15. Van der Linden JCS, Guimarães, LBM. Diferenças de gênero na percepção de riscos relacionados ao calçado feminino de salto alto e bico fino. Anais do VI Congresso Internacional de Ergonomia e Usabilidade de Interfaces Humano-Tecnologia: produtos, informação, ambiente construído, transporte; Bauru. Bauru: UNESP; 2006. p. 7.

16. Cair de salto alto é acidente de trabalho, decide Justiça. 2005. [acesso 6 nov. 2007] Disponível em: http:// tsnogueira. blogspot.com/2010/04/o-perigo-da-salto-alto-na-empresa.html

17. Anjos L. Body mass index as a tool in the nutritional assessment of adults: a review. Rev Saúde Públ. 1992;26(6):431-6.

18. Gajdosik R, Bohannon R. Clinical measurement of range of motion. Review of goniometry emphasizing reliability and validity. Phys Ther. 1987;67(12):1867-72.

19. Rothstein J, Miller P, Roettger R. Goniometric reliability in a clinical setting. Elbow and knee measurements. Phys Ther. 1983;63(10):1611-15.

20. Braz R, Goes F, Carvalho G. Confiabilidade e validade de medidas angulares por meio do software para avaliação postural. Fisioter Mov. 2008;21(3):117-26.

21. Ferreira E. Postura e controle postural: desenvolvimento e aplicação de método quantitativo de avaliação postural. [tese]. São Paulo: Universidade de São Paulo; 2006.

22. Young M. A review on postural realignment and its muscular and neural components. 2003. [acesso 21 fev. 2010]. Disponível em: http://www.elitetrack.com/article_files/posture.pdf

23. Oliver J, Middleditch A. Anatomia funcional da coluna vertebral. Rio de Janeiro: Revinter; 1998.

24. Hansen A, Childress D. Effects of shoe heel height on biologic rollover characteristics during walking. J Rehabil Res Dev. 2004;41(4):547-54.

25. Oliveira S, Santos M, Santos G, Monteiro W, Oliveira C, Fregni F. Análise do tempo de adaptação da marcha em mulheres adultas jovens. Anais do X Encontro Latino Americano de Iniciação Científica e VI Encontro Latino Americano de Pós-Graduação - Universidade do Vale do Paraíba; São José dos Campos, SP: UNIVAP; 2006. p. 584-587.

26. Melo F. Controlo postural: controlo reflexo versus controlo dinâmico. Rev Bras Educ Fís Esp. 2006;20(s5):107-09.

27. Bankoff A, Ciol P, Zamai C, Schmidt A, Barros D. Estudo do equilíbrio corporal postural através do sistema de baropodometria eletrônica. Conexões. 2004;2(2):87-104.

28. de Luca C. Low Back Pain: A major problem with low priority. J Rehabil Res Dev. 1997;34(4):7-8.

29. Gefen A, Megido-Ravid M, Itzchak Y, Arcan M. Analysis of muscular fatigue and foot stability during high-heeled gait. Gait \& Posture. 2002;15(1):56-63.

30. Stokes I, Allard P, Blachi J. Three dimensional analysis of human movement. Champaign: Human Kinetics Publishers; 1995.

31. Casarin C. A influência do calçado de salto alto sobre a lordose lombar associada aos músculos lombares e gastrocnêmio. 2005. [dissertação]. São Paulo: Universidade Estadual de Campinas, Faculdade de Odontologia de Piracicaba; 2005.

32. Anderson J. Cognitive skills and their acquisition. Hillsdale, NJ: Lawrence Erlbaum; 1981.

Recebido: 25/03/2009

Received: 03/25/2009

Aprovado: 20/05/2010

Approved: 05/20/2010 